\title{
ANÁLISE DA SITUAÇÃO DA POLIOMIELITE EM UMA AMOSTRA DE CRIANÇAS DA CIDADE DE SÃO PAULO, COM O AUXILIO DE UM PARÂMETRO DE VIGILÂNCIA EPIDEMIOLÓGICA.
}

Klaus E. Stewien* Victório Barbosa**

STEWIEN, K.E. \& BARBosa, V. Análise da situação da poliomielite em uma amostra de crianças da cidade de São Paulo, com o auxillo de um parâmetro de vigilância epidemiológica. Rev. Saúde públ., S. Paulo, 11:338-44, 1977.

REsumo: A situação da poliomielite é analisada em uma amostra de crianças de 0-10 anos de idade da cidade de São Paulo, assistidas pelo Hospital Menino lesus, com o auxilio de um parâmetro de vigilância epidemiológica - o fator $r$ da dinâmica da imunização. Os resultados de um recente inquérito soro-epidemiológico foram transformados matematicamente na curva de crescimento expressa pela função exponencial $y=a+(1-a)(1-r t)$, aumentando-se, com isto, consideravelmente $o$ valor informativo do inquérito. A análise mostrou que pela vacinação oral se imunizaram efetivamente cerca de $50 \%$ e $60 \%$ do total dos individuos da população infantil de 0-10 anos de idade contra os poliovirus dos tipos 1 e 2, respectivamente. Além disto, houve em decorrệncia da circulação de poliovirus, nesta população, em média, cerca de 85 infeccóes com poliovirus do tipo 1 e 70 infeç̧óes com o poliovirus do tipo 2, por 1.000 habitantes, por ano. Tendo em vista que o coeficiente de morbidade da poliomielite alcança cifras da ordem 31 e 27 casos por 100.000 habitantes, respectivamente nos grupos etários de $0-1$ e $1-2$ anos de idade, os autores recomendam que não sejam poupados esforços no sentido de que as crianças sejam efetivamente vacinadas dentro do esquema de imunização estabelecido, recebendo a $1^{a}$ dose da vacina já aos 2 oll 3 meses de idade.

Unitermos: Poliomielite, São Paulo, Brasil. Vigilância epidemiológica. Inquérito soro-epidemiológico. Vacina Sabin.

\section{INTRODUÇÃO}

A apresentação dos resultados de inquéritos soro-epidemiológicos tem sido tradicionalmente feita por meio de freqüências relativas de indivíduos imunes da população estudada. Entretanto, tal procedimento nāo mostra o total conteúdo das informações obtidas nestas investigações. Assim, por exemplo, não permite conhecer a dinâmica segundo a qual ocorrem infeç̧óes numa população, perdendo-se a importante infor-

* Do Departamento de Microbiologia e Imunologia do Instituto de Ciências Biomédicas da USP - "Setor Saúde Pública" - Av. Dr. Arnaldo, 715 - São Paulo, SP - Brasil. Do Departamento de Epidemiologia da Faculdade de Saúde Pública da USP - Ar. Dr. Arnaldo, 715 - São Paulo, SP - Brasil. 
STEWIEN. K. E. \& BARBOSA, V. Análise da situação da poliomielite em uma amostra de crianças da cidade de São Paulo, com $o$ auxílio de um parâmetro de vigilância epidemiológica. Rev. Saúde públ., S. Paulo, 11:338-44, 1977.

mação a respeito do número de individuos que anualmente entram em contato com um determinado agente patogênico.

Sendo os resultados obtidos en inquéritos sorológicos transformados matematicamente em curva de crescimento, com função exponencial $y=1-\mathrm{e}^{-\mathrm{r} 1}$, é possivel medir as forças de infecção que atuam sobre os individuos succeptiveis de uma população ${ }^{2}, 3,{ }^{\circ}$.

A constante " $r$ " determina a proporção de individuos que anualmente, em média, tiveram contato efetivo com um determinado agente infeccioso e se imunizaram. Desta forma, os valores numéricos das percentagens de imunes podem ser reduzidos na referida expressão matemática universal.

A utilização da constante " $r$ " como parâmetro de vigilância epidemiológica é, sem dúvida, de grande valor prático para a saúde pública, visto que ela permite evidenciar lacunas na imunidade de populaçòes em estudo e, conseqüentemente, tomar, em tempo hábil, as medidas de prevenção indicadas.

Além disso, essa constante " $r$ " é uma grandeza pouco influenciada pelo tamanho da amostra de individuos examinados e independente da amplitude dos grupos etários constituidos ${ }^{3}, 6$. Também não é influenciada por pequenas variações de sensibilidade da técnica sorológica empregada, o que possibilita a comparação dos resultados de inquéritos soro-epidemiológicos conduzidos em laboratórios distintos, bem como de inquéritos realizados em áreas geográficas e em épocas diferentes. ${ }^{6}$

O objetivo do presente trabalho é o de analisar a situação epidemiológica da poliomielite na Capital de São Paulo, mediante a utilização do parâmetro antes descrito, com base nos resultados obtidos em recente inquérito soro-epidemiológico realizado nessa Capital 1.

\section{MATERIAL E METODOS}

\section{Grupo de crianças estudadas}

O grupo em estudo é representado por uma amostra casual de uma população de fácil acesso, constituída de crianças de 0-10 anos de idade, predominantemente da classe pobre do Município de São Paulo, que aflui, em qualquer época, ao Hospital Menino Jesus, da Prefeitura Municipal. As crianças, em número de 720 , eram procedentes de todos os distritos e subdistritos da Capital de São Paulo ${ }^{1}$.

\section{Inquérito sorológico}

Os métodos utilizados para a determinação dos anticorpos neutralizantes contra os poliovírus dos tipos 1,2 e 3 foram anteriormente descritos (Barbosa \& Stewien', 1975). Em resumo, os soros de 720 crianças foram examinados na diluição de $1: 10$, segundo a microtécnica, utilizando-se as estirpes de referência Mahoney, MEF-1 e Saukett.

\section{Parâmetro de vigilância epidemiológica - Fator " $r$ " da dinâmica de imunização}

Este parâmetro caracteriza o estado imunitário da população em estudo, pois determina a proporção de individuos que tiveram contato com o vírus da poliomielite e, desta forma, através da formação de anticorpos protetores, adquiriram imunidade. A dinâmica dessa imunização segue essencialmente as regras da curva de crescimento, expressa pela função exponencial $y=1-e^{-r t}$, que apresenta, no gráfico que a representa, nas ordenadas $y$ a proporção de indivíduos imunes e nas abcissas $t$ a idade dos indivíduos examinados. Como no caso particular do estudo realizado por Barbosa \& Stewien, 1975ㄹ, a transmissão endêmica da poliomielite não representava a única força de infecção que atuou sobre o grupo de crianças estudado, uma vez 
STEWIEN, K. E. \& BARBOSA, V. Análise da situação da poliomielite em uma amostra de crianças da cidade de São Paulo, com o auxilio de um parâmetro de vigilância epidemiológica, Rev. Saúde públ., S. Paulo. 11:338-44, 1977.

que a maioria das crianças tinha recebido a vacina Sabin, o modelo matemático que melhor se aplicada ao referido caso é aquele representado pela função:

$$
y=a+(1-a)\left(1-e^{-r t}\right),
$$

em que $a$ representa a fração de individuos que sofrem os efeitos drásticos da vacinação oral e $r$, a proporção que se infectou anualmente com poliovirus circula intes na comunidade. Os valores de $a$ e $r$ foram estimados mediante utilização de um nomograma, segundo Muench : e Thraenhart e col. ${ }^{*}$, após transformação matemática dos resultados do inquérito sorológico realizado.

\section{RESULTADOS E DISCUSSAOO}

A Tabela 1 apresenta, respectivamente para os poliovirus dos tipos 1 e 2 , os resultados do inquérito realizado na cidade de São Paulo', assim como a transformação matemática destes resultados e o cálculo dos pontos da curva exponencial de crescimento. A tabela mostra que o grupo etário menos protegido contra a poliomielite é o das crianças do primeiro ano de vida, onde as taxas de positividade alcançam apenas $40,6 \%$ para o tipo 1 e $48,6 \%$ para o tipo 2. Ao contrário, o grupo etário de 6-10 anos apresenta o maior indice de proteção com taxas de $75,3 \%$ e $74,6 \%$, respectivamente. Deixamos de apresentar os resultados referentes ao tipo 3 porque não obedeceram as regras da curva de crescimento.

$\mathrm{Na}$ Tabela 2 temos a distribuição etária dos casos de poliomielite ocorridos no ano do inquérito (1973), bem como as frequiências acumuladas destes casos e os coeficientes de morbidade por grupo etário (por cem mil habitantes). Verificamos na tabela que os coeficientes são particularmente elevados nos grupos etários de 0-1 e 1-2 anos, nos quais se acumulam $75,4 \%$ dos casos paraliticos ocorridos.
As Figuras 1 e 2 apresentam, respectivamente para os poliovírus dos tipos 1 e 2 , em forma de histograma, as proporções de crianças imunes, por grupo etário, bem como as curvas de crescimento decorrentes da transformação matemática dos resultados obtidos no inquérito. As funções exponenciais que deram origem às curvas apresentadas são respectivamente para os poliovirus dos tipos 1 e 2 :

$$
\begin{aligned}
& Y_{1}=0,51+0,49(1-\mathrm{e}-0,0851) \\
& Y_{2}=0,59+0,41(1-\mathrm{e}-\mathrm{e},-0,0701)
\end{aligned}
$$

Nestas fórmulas os valores estimados para $a$ e $r$ foram, respectivamente, 0,51 e 0,085 (tipo 1) e 0,59 e 0,070 (tipo 2). Os valores de a mostram que a vacina Sabin aplicada pelo programa de vacinação antipoliomielitica, até então desenvolvido no Município de São Paulo, imuniza anualmente, em média, cerca de $50 \%$ e $60 \%$ dos individuos contra os poliovírus dos tipos 1 e 2 , respectivamente, niveis estes que, entretanto, estão aquém do desejável para efetivo controle da doença, ou seja, de $75 \%{ }^{1}$. Possivelmente, estes valores também tenham sido influenciados pela ocorrência de um surto epidêmico de poliomielite no Município de São Paulo, nos primeiros meses de 1971. Já os valores de $r$ indicam, em termos práticos, que a população estudada sofreu anualmente, em média, neste periodo, 85 infecçōes com o poliovirus do tipo 1 e 70 infecções com o poliovirus do tipo 2 , por nil habitantes, decorrentes da transmissão endêmica da poliomielite entre nós, ainda presente porque, como vimos, o programa de vacinação referido não foi suficiente para interromper definitivamente a circulação de poliovírus na nossa comunidade.

Se realizarmos uma análise comparativa entre os histogramas das Figuras 1 e 2 e as suas respectivas curvas de crescimento, vericaremos que os valores dos histogramas 
STEWIEN, K. E. \& BARBOSA, V. Análise da situação da poliomielite em uma amostra de crianças da cidade de São Paulo, com 0 auxílio de um parâmetro de rigilância epidemiológica. Rev. Saúde públ., S. Paulo, 11:338-44, 1977.

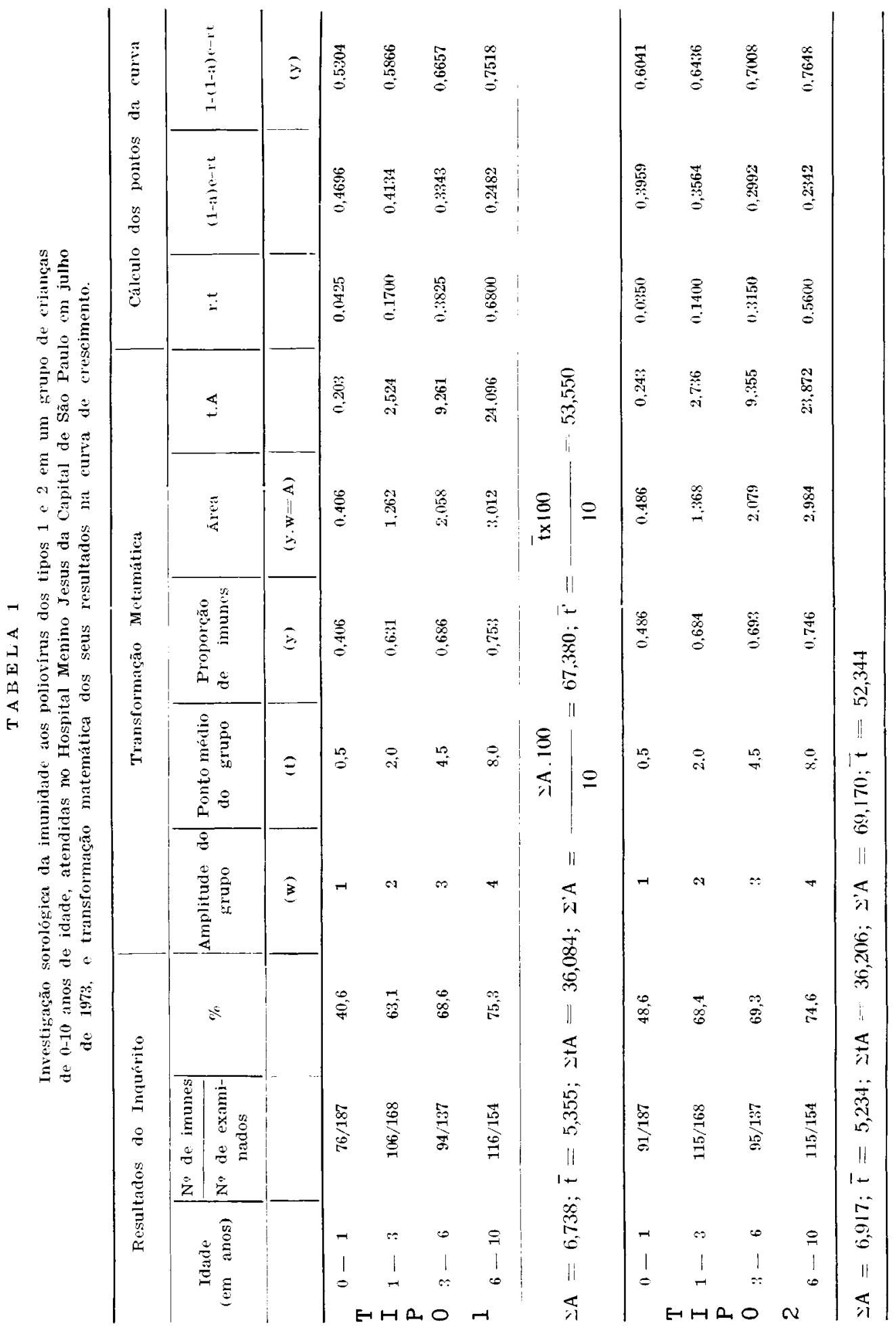


STEWIEN, K. E. \& BARBOSA. T. Análise ia situação da poliomielite em uma amostra de crianças da cudade de São Paule, $c o m$ a auxílı de um parâmetro de vigilancia epudemiológiea. Rer. Saúde públ., S. Paulo, 11:338-44, $197 \pi$.

T A B E L A 2

Distribuição etária do número de casos de poliomielite, da freqüência acumulada e do coeficiente de morbidade, no Município de São Paulo - 1973.

\begin{tabular}{c|c|c|c|c}
\hline $\begin{array}{c}\text { Idade } \\
\text { em anos) }\end{array}$ & $\begin{array}{c}\text { Número de } \\
\text { casos }\end{array}$ & $\begin{array}{c}\text { Número de } \\
\text { casos acumu- } \\
\text { ladios }\end{array}$ & $\%$ & $\begin{array}{c}\text { Coeficiente } \\
\text { de morbi- } \\
\text { dade* }\end{array}$ \\
\hline-1 & 48 & 48 & 42,1 & 31,6 \\
1 & 38 & 86 & 75,4 & 27,5 \\
2 & 14 & 100 & 87,7 & 9,3 \\
3 & 5 & 105 & 92,1 & 1,9 \\
$\mathbf{4}$ & 3 & 108 & 94,7 & 1,2 \\
5 & 2 & 110 & 96,5 & 1,3 \\
7 & 2 & 112 & 98,2 & - \\
8 & 1 & 112 & 98,2 & 0,7 \\
$9-14$ & - & 113 & 99,1 & 0,7 \\
15 & 1 & 114 & 100,0 & 1,7 \\
\hline TOTAL & 114 & 114 & 100,0 & \\
\hline
\end{tabular}

* (por 100.000 habitantes)

Fonte: Departamento Regional de Saúde da Grande São Paulo

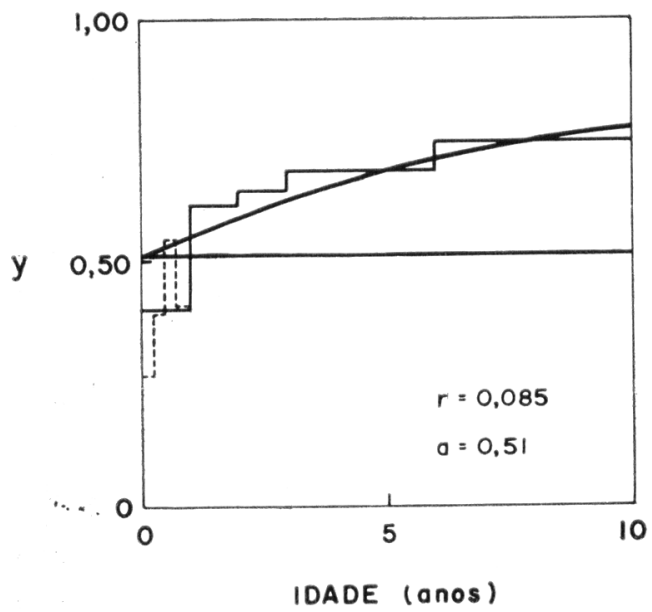

Flg. 1 - Estado imunitário contra o poliovírus do tipo 1 de um grupo de crianças de $0-10$ anos de jdade atendidas no Hospital Menino Jesus da Prefeitura do Município de São Paulo em julho de 1973, apresentado em histograma - em curva de crescimento da dinamica da imunização $y=a+(1-a)(1-e-r 1)$.

do grupo etário de 0-1 ano ficam bem abaixo dos valores das curvas, passando a se situarem acima dos pontos das curvas nos grupos etárius seguintes. Isto significa

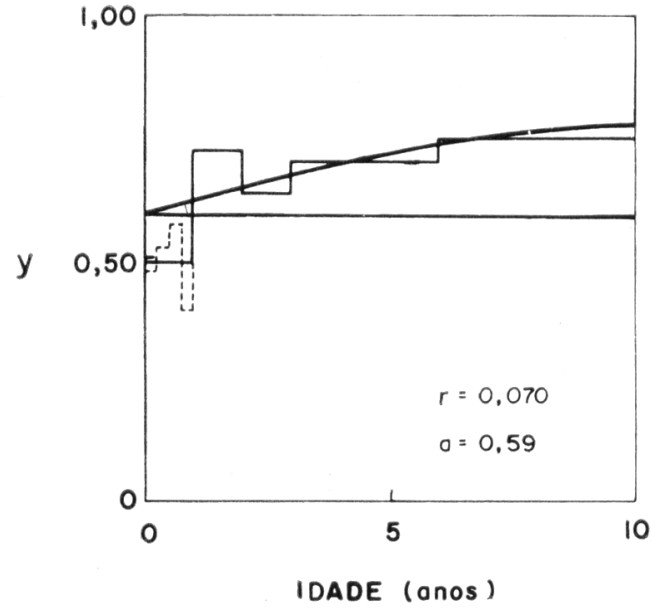

Fig. 2 -- Estado imunitário contra o poliovírus do tipo 2 de un grupo de crianças de $0-10$ anos de idade, atendidas pelo Hospital Menino Jesus da Prefeitura do Município de São Paulo em julho de 1973, apresentado em histograma e em curra de crescimento da dinamica da imunização $y=a+(1-a)(1-e-1)$.

que existe um aumento acentuado no numero de infecçóes durante a passagem do 10 para o 2 ano de vida das crianças do grupo estudado. A explicação deste feno- 
STEWIEN, K, E. \& BARBOSA, V. Análise da situação da poliomílite em uma amostra de crianças da cidade de Săo Paulo, com $ڤ$ auxílio de um parâmetro de vigilancia epidemiológica. Rev. Saúde públ., S. Paulo, 11:338-44, 1977.

meno reside na queda dos anticorpos maternos contra a poliomielite, presentes nas crianças, e conseqüente aumento de susceptibilidade à infecção. Que as infecções sejam devidas a transmissão endêmica da poliomielite, também mostrou os coeficientes de morbidade particularmente elevados nos grupos etários de 0-1 e 1-2 anos, (Tabela 3), atingindo as elevadas cifras de 3,6 e 27,5 casos em cem mil habitantes respectivamente.

Disto se conclui que o programa de imunização não conseguiu interromper a circulação natural de poliovirus em nosso meio.

Em face desta situação da poliomielite na capital de São Paulo, todos os esforços devem ser unidos no sentido de que, na prática da vacinação oral, as crianças recebam a primeira dose de vacina Sabin já aos 2 ou 3 meses de idade, o que impediria a formação de grupos populacionais susceptiveis à poliomielite.

STEWIEN, K. E. \& BARBosa, V. /Analysis of the poliomyelitis situation in the city of S. Paulo, Brazil by means of an epidemiologic surveillance parameter] Rev. Saúde públ., S. Paulo, $11: 338-44,1977$.

ABSTRACT: The sifuation of poliomyelitis in the city of São Paulo is analysed by means of an epidemiologic surveillance parameter - the " $r$ " factor of immunization events. The results of a recent sero-epidemiologic survey realized in this City were transformed into the catalytic growth curve $y=a+(1-a)$ $\left(1-e^{-r t}\right)$. The informative value of the survey is therefore considerably enhanced. The analysis showed that about $50 \%$ and $60 \%$ of the population of children 0-10 years old were immunized by oral polio vaccination against type 1 and 2 polioviruses, respectivilly-Further, the average rate of effective contacts between individuals of the entire population and type 1 and 2 polioviruses were, per annum, respectively 85 and 70 per 1000 inhabitants. Due to the relative high mortality rate to poliomyelitis for children of the first year of life (31 cases per $100.000)$, it is emphasized that children must receive oral vaccine at 2 or 3 month of age.

Uniterms: Poliomyelitis, S. Paulo, Brazil. Epidemiologic surveillance. Seroepidemiologic survey. Poliovirus vaccine, oral.

\section{REFERENCIAS BIBLIOGRAFICAS}

1. BARBosA, V. \& STEWIEN, K.E. Estado imunitário relativo à poliomielite das crianças de $0-12$ anos, residentes no Municipio de São Paulo, Brasil e assistidas pelo Hospital Menino Jesus. Rev. Saúde públ., S. Paulo, 9:137-53, 1975 .
2. MUENCH, H. Derivation of rates from summation data by the catalytic curve. J. Amer. statist. Ass., 29 :2538, 1934.

3. MUENCH, H. Catalytic models in epidemiology. Cambridge, Mass. Harvard University Press, 1959 
STEWIEN, K. E. \& BARBOSA, V. Análise da situação da poliomielite em uma amostra de crianças da cidade de São Paulo, com o auxílio de um parâmetro de vigilância epidemiologica. Rev. Saúde públ., S. Paulo, 11:338-44, 1977.

4. RAMOS-ALVAREZ, M. Poliomyelitis. In: INTERNACIONAL CONFERENCE ON VACCINES AGAINST VIRAL AND RICKETTSIAL DISEASES OF MAN, 1st, Washington, D.C., 1966. Washington, D.C., Pan American Health Organization, 1967. p. 213-4. (PAHO Scient. publ, , 147).

5. SWARTZ, T.A. et al. Non-poliovirus interference with oral polio vaccine immunization: possible influence of physical climate and socio-economic status.
Advanc. exp. med. Biol., 31:237-40, 1972.

6. THRAENHART, $O$. et al. Der epidemiologische Aussagewert der katalytischen Wachstumskurve für Populations - Immunität und Impfindikation am Beispiel einer Polio Typ 1 - Epidemie im Ruhrgebiet. Z. med. Mikrobiol. Immunol., 156:175-93, 1971.

Recebido nara publicação em 07/03/1977 Aprovado nara publicaça em 28/03/197\% 